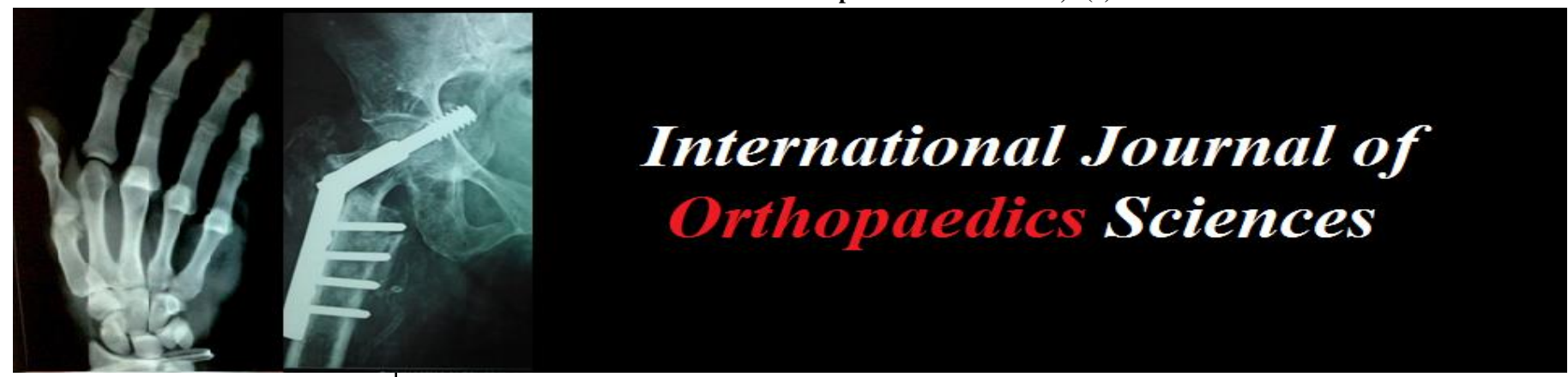

ISSN: $2395-1958$

IJOS 2018; 4(3): 542-546

(C) 2018 IJOS

www.orthopaper.com

Received: 07-05-2018

Accepted: 08-06-2018

Mukesh Phalak

Associate Professor, Department of Orthopaedics, Dr D. Y. Patil

Medical College, Pimpri, Pune,

Maharashtra, India

\section{Nitin Wadhwa}

Junior Resident, Department of Orthopaedics, Dr D. Y. Patil Medical College, Pimpri, Pune, Maharashtra, India

\section{Clevio Desouza}

Junior Resident, Department of Orthopaedics, Dr D. Y. Patil Medical College, Pimpri, Pune, Maharashtra, India

\section{Shiju George}

Junior Resident, Department of Orthopaedics, Dr D. Y. Patil

Medical College, Pimpri, Pune,

Maharashtra, India

\section{Correspondence}

Mukesh Phalak

Associate Professor, Department of Orthopaedics, Dr D. Y. Patil Medical College, Pimpri, Pune,

Maharashtra, India

\section{Effect of distraction osteogenesis in patient with tibial shortening after initial union of congenital pseudarthrosis of the tibia (CPT)}

\author{
Mukesh Phalak, Nitin Wadhwa, Clevio Desouza and Shiju George
}

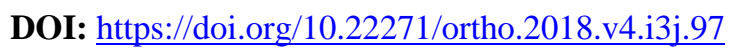

\section{Abstract}

Background: This is a Retrospective study which was conducted with the purpose of evaluating the preliminary result of distraction osteogenesis in patients with tibial shortening after initial union of Congenital Pseudarthrosis of the Tibia (CPT).

Methods: Identification of all the CPT cases with tibial shortening after initial union managed by proximal tibia lengthening using Ilizarov technique was done. All the patients charts and radiograms were reviewed.

Results: 11 CPT cases were included between March 2013 and January 2018 with an average follow up of 41 months (range, 34-51 months). 8.5 years was the mean age at surgery. The average length of discrepancy was $5.6 \mathrm{~cm}$ (range, 2.0-8.2 cm). Radiological findings of proximal tibial dysplasia were found in eight cases while three other cases had no radiological findings. The average distraction length gained was $5.3 \mathrm{~cm}$ (range, $3.5-8.0 \mathrm{~cm}$ ) with a mean elongation rate of $21.4 \%$ (range, $15-30 \%$ ). The Healing Index (HI) was $63.1 \mathrm{~d} / \mathrm{cm}$ (range, $47-77 \mathrm{~d} / \mathrm{cm}$ ). The Findings seen in the eight patients with proximal tibial dysplasia was that out of eight cases, five cases had lateral callus, three had central callus and poor bone regeneration was seen in all of them. In the three patients who had no radiological findings concave shaped callus was identified with an average HI of $52.7 \mathrm{~d} / \mathrm{cm}$. None of the patients had refracture, non-union, axis deviation or angulation of the distraction area. Two of the patients were found to have ankle stiffness. There was no evidence of knee contracture in any of the patients. There were five cases with pin tract infection which was managed by regular dressing and oral antibiotics.

Conclusions: We concluded that proximal tibial lengthening after initial union of CPT was effective for management of tibial shortening, however poor bone regeneration with various types of callus from normal, greater healing index and prolonged fixator wearing was seen. We recommended that when the limb length discrepancy (LLD) was more than $4 \mathrm{~cm}$ in younger children after primary union of CPT, tibia lengthening could be considered.

Keywords: Pseudarthrosis, tibia, distraction, osteogenesis

\section{Introduction}

Congenital pseudarthrosis of the tibia (CPT) is one of the most difficult orthopaedics problems to manage in world. The occurrence rate of CPT is 1 in 190,000 live births ${ }^{[1]}$. However, with developments of techniques like the combined surgery of Ilizarov device, bone grafting and intramedullary fixation, there has been an increase in the primary bone union rate to $86 \%$ [2-5]. Surgical intervention is still required however for deformities such as limb length discrepancy, mechanical axis deviation, joint stiffness and re-fracture ${ }^{[6]}$. The most frequently encountered problem during the treatment of CPT is Tibial shortening ${ }^{[7,8]}$. There are three methods to manage tibial shortening which include lengthening of the short bone, arresting the growth of the contralateral leg or a combination of both the procedures. Among the three methods, lengthening of the affected side is the most commonly used method. Cho et al. ${ }^{[9]}$ reported their experience of proximal tibial lengthening by distraction osteogenesis in CPT. Bone transport with the lengthening through the physis in CPT patients to equalize limb length discrepancy was described by Vlad et al. ${ }^{[10]}$. However, there were few reports on the indication and time window for tibial lengthening on CPT children ${ }^{[11]}$.

Through this paper we have presented our experiences of 11 CPT cases with proximal tibial lengthening using Ilizarov technique after initial union of tibial pseudarthrosis and evaluated 
its outcome. We aimed to describe the clinical features of tibial lengthening in CPT patients and provide guidance to such procedures.

\section{Methods}

This was a retrospective study. A written informed consent was obtained from all the patients or guardians. Consent to publication of their medical data including photographs and images from all the guardians and patients were also obtained. Identification of all the CPT cases with tibial shortening after initial union which were managed by proximal tibial lengthening using Ilizarov technique was done. Review of all the patient charts and radiographs was done. Between March 2013 and January 2018, 11 CPT cases were included. Ten males and one female with four cases of the right side and seven of the left were included in the study. A combined Ilizarov fixator with intramedullary fixation of the tibia and wrapping autogenic iliac bone graft technique was used to obtain initial union in all the cases.

According to Ohnishi $X$ ray evaluation criteria ${ }^{[12]}$, all the cases had primary union of CPT more than 2 years before distraction osteogenesis. There was association of ten patients with type I Neurofibromatosis (NF-1). The mean age at surgery was 8.5 years (range, 3.9-14.5y). The average length of discrepancy was $5.6 \mathrm{~cm}$ (range, $2.0-8.2 \mathrm{~cm}$ ).

Eight out of eleven cases had radiological findings of proximal tibial dysplasia, out of which 1 case had trumpetshaped narrowing of proximal tibia metaphysis, 4 cases had anterior inclination of the proximal tibial physis and 3 cases had concavity of the anterior cortex. In addition, there was 1 case with $20^{\circ}$ and $15^{\circ}$ angulations of tibia in AP and lateral view of radiographies (Patient 4). None of the cases had tibial lengthening before the procedure. The data was shown in Table 1.

Table 1: Patient data and results

\begin{tabular}{|c|c|c|c|c|c|c|c|c|}
\hline $\begin{array}{c}\text { Case } \\
\text { no. }\end{array}$ & Gender & $\begin{array}{c}\text { Age, } \\
\mathbf{y}\end{array}$ & $\begin{array}{c}\text { Associated } \\
\text { NF1 }\end{array}$ & $\begin{array}{c}\text { Proximal tibial } \\
\text { dysplasia }\end{array}$ & $\begin{array}{c}\text { LLD, } \\
\mathbf{c m}\end{array}$ & $\begin{array}{c}\text { Length gain, } \\
\mathbf{c m}\end{array}$ & $\begin{array}{c}\text { Elongation } \\
\text { rate, } \%\end{array}$ & $\begin{array}{c}\text { HI, } \\
\mathbf{d} / \mathbf{c m}\end{array}$ \\
\hline 1 & male & 11.9 & yes & yes & 7.0 & 7.0 & 25 \\
\hline 2 & female & 3.9 & yes & yes & 5.0 & 5.0 & 21 \\
\hline 3 & male & 8.7 & yes & yes & 4.0 & 4.0 & 62 \\
\hline 4 & male & 4.9 & no & yes & 2.0 & 3.5 & 18 & 16 \\
\hline 5 & male & 4.1 & yes & yes & 3.5 & 3.8 & 76 \\
\hline 6 & male & 14.5 & yes & yes & 8.0 & 8.0 & 22 & 30 \\
\hline 7 & male & 6.8 & yes & no & 6.0 & 6.0 & 63 \\
\hline 8 & male & 9.2 & yes & yes & 5.0 & 5.0 & 68 \\
\hline 9 & male & 12.0 & yes & yes & 8.2 & 4.2 & 19 & 15 \\
\hline 10 & male & 11.3 & yes & no & 8.0 & 6.5 & 70 \\
\hline 11 & male & 6.2 & yes & no & 4.5 & 5.2 & 64 \\
\hline Average & NA & 8.5 & NA & NA & 5.6 & 5.3 & 25 \\
\hline
\end{tabular}

NA Not applicable

All the surgeries in this study were performed by a senior orthopaedic surgeon. The procedure respected the principles of Ilizarov external frame application. The Ilizarov's fixator was installed with one set of 2 rings above the proximal tibia, 1 set of 2 rings below the proximal tibia and 1 set of 2 rings in the distal tibia. Bone segments proximal and distal to the corticotomy site were fixed with at least 3 pins. Then corticotomy of proximal tibia was done under fluoroscopy. A special preassembled orthosis was connected to the proximal ring of the Ilizarov fixator in order to prevent knee contracture during the lengthening period (Fig. 1). The "lock" on the orthosis would keep the patient in a knee-extension position for 8 hours a day, however, the "hinge" allowed free movement of knee joint during the other 16 hours a day.

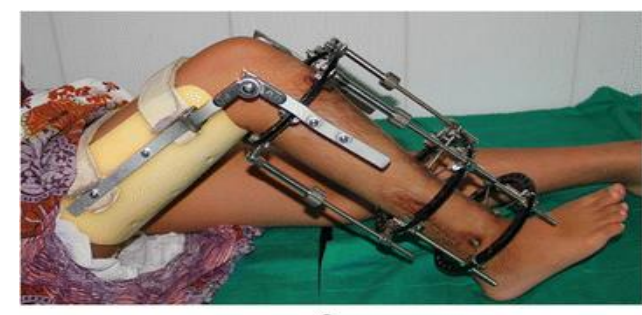

a

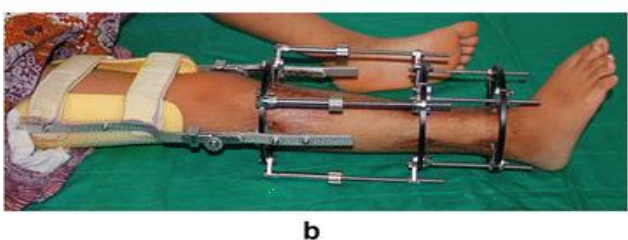

Photograph of patient wearing knee orthosis $(a, b)$
One week post operatively, distraction was carried and the speed for bone transportation procedures was adjusted to $0.5 \mathrm{~mm} /$ day in four increments. For cases whose intramedullary nailing was in the tibia, passive and active ankle dorsiflexion was carried out three times per day to prevent equinus deformity. The radiographs were taken ten days after distraction, then in a monthly interval, to observe the osteotylus quality and the alignment of tibia. The distraction rate was adjusted according to the radiographic features of distraction osteogenesis with regard to osteotylus shape described by $\mathrm{Ru} \mathrm{Li}$ et al. ${ }^{[13]}$. If the osteotylus shape was fusiform or cylindrical, a distraction rate of $0.5 \mathrm{~mm} /$ day was continued. While a concave shaped callus was discovered on X-rays, then the distraction rate should adjusted to $0.25 \mathrm{~mm} /$ day. When the shape was lateral or central, the distraction should be suspended for 2 weeks until new regenerate bone formation was observed around the lateral or central shaped callus, however the rate should be $0.25 \mathrm{~mm} /$ day when we restarted the distraction. The criterion used for Ilizarov fixator removal was regard as the formation of more than 3 cortices across the distraction gap on AP and lateral X-rays ${ }^{[6]}$. When the fixator was removed, a long leg cast was applied for $6-8$ weeks followed by a protective long leg orthosis until skeletal maturity. Healing index (HI) was calculated as the number of days necessary to lengthen and consolidated $1 \mathrm{~cm}{ }^{[9]}$.

\section{Results}

11 CPT cases were included between March 2013 and January 2018 with an average follow up of 41 months (range, 34-51 months). 8.5 years was the mean age at surgery. The 
average length of discrepancy was $5.6 \mathrm{~cm}$ (range, $2.0-8.2 \mathrm{~cm}$ ). Radiological findings of proximal tibial dysplasia were found in eight cases while three other cases had no radiological findings. The average distraction length gained was $5.3 \mathrm{~cm}$ (range, $3.5-8.0 \mathrm{~cm}$ ) with a mean elongation rate of $21.4 \%$ (range, 15-30\%). The Healing Index (HI) was $63.1 \mathrm{~d} / \mathrm{cm}$ (range, 47-77 d/cm). The Findings seen in the eight patients with proximal tibial dysplasia was that out of eight cases, five cases had lateral callus, three had central callus and poor bone regeneration was seen in all of them. In the three patients who had no radiological findings concave shaped callus was identified with an average HI of $52.7 \mathrm{~d} / \mathrm{cm}$. None of the patients had refracture, non-union, axis deviation or angulation of the distraction area. Two of the patients were found to have ankle stiffness. There was no evidence of knee contracture in any of the patients. There were five cases with pin tract infection which was managed by regular dressing and oral antibiotics. The outcome of a case is presented in Figure no 2 and 3.
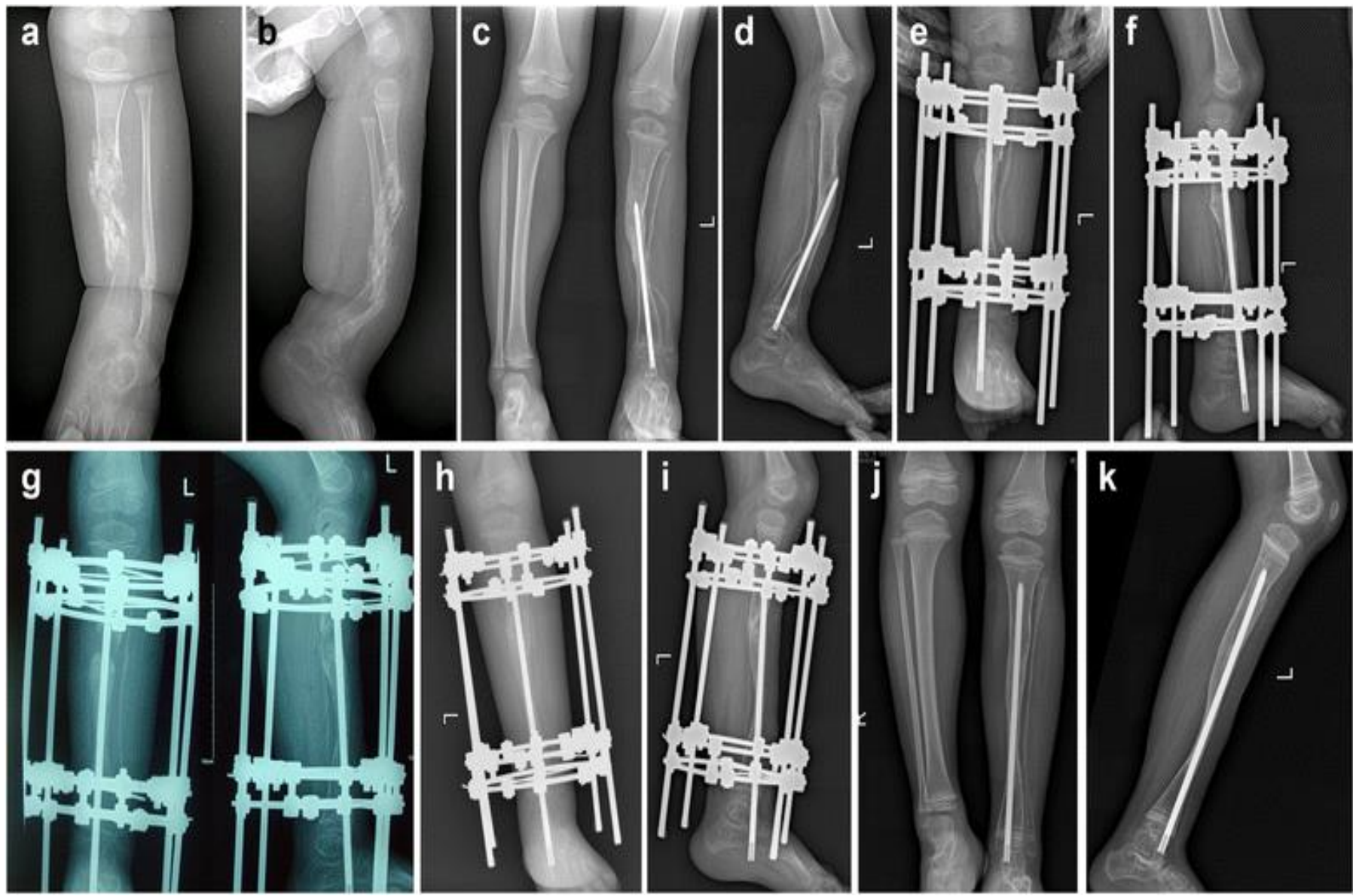

Fig 2

A 2.1y boy (Patient 4) with CPT of the left had two procedures of the tibia in another hospital previously. Radiographs showed the fragmental bone grafting but nonunion of the pseudarthrosis $(a, b)$. Two years after combined surgery with Ilizarov fixator, intramedullary rodding of the tibia and wrapping autogenic iliac bone graft, the radiography showed a primary union of pseudarthrosis with LLD of $2 \mathrm{~cm}$ and angulation of tibia (c, d). A proximal tibial osteotomy and lengthening was carried out to correct the angulation deformity and equalize the limb length (e, f). Lateral callus was observed in this patient $(\mathrm{g})$. Lengthening procedure was suspended for 2 weeks until new regenerate bone formation was observed around the callus. The length gained was $3.8 \mathrm{~cm}$ with $\mathrm{HI}$ of $63 \mathrm{~d} / \mathrm{cm}(\mathrm{h}, \mathrm{i})$. X rays of the last follow-up showed solid union of the lengthening segment and good alignment of tibia $(\mathrm{j}, \mathrm{k})$. The next step is to push the whole rod into tibia cavity when there is enough length in proximal tibia 

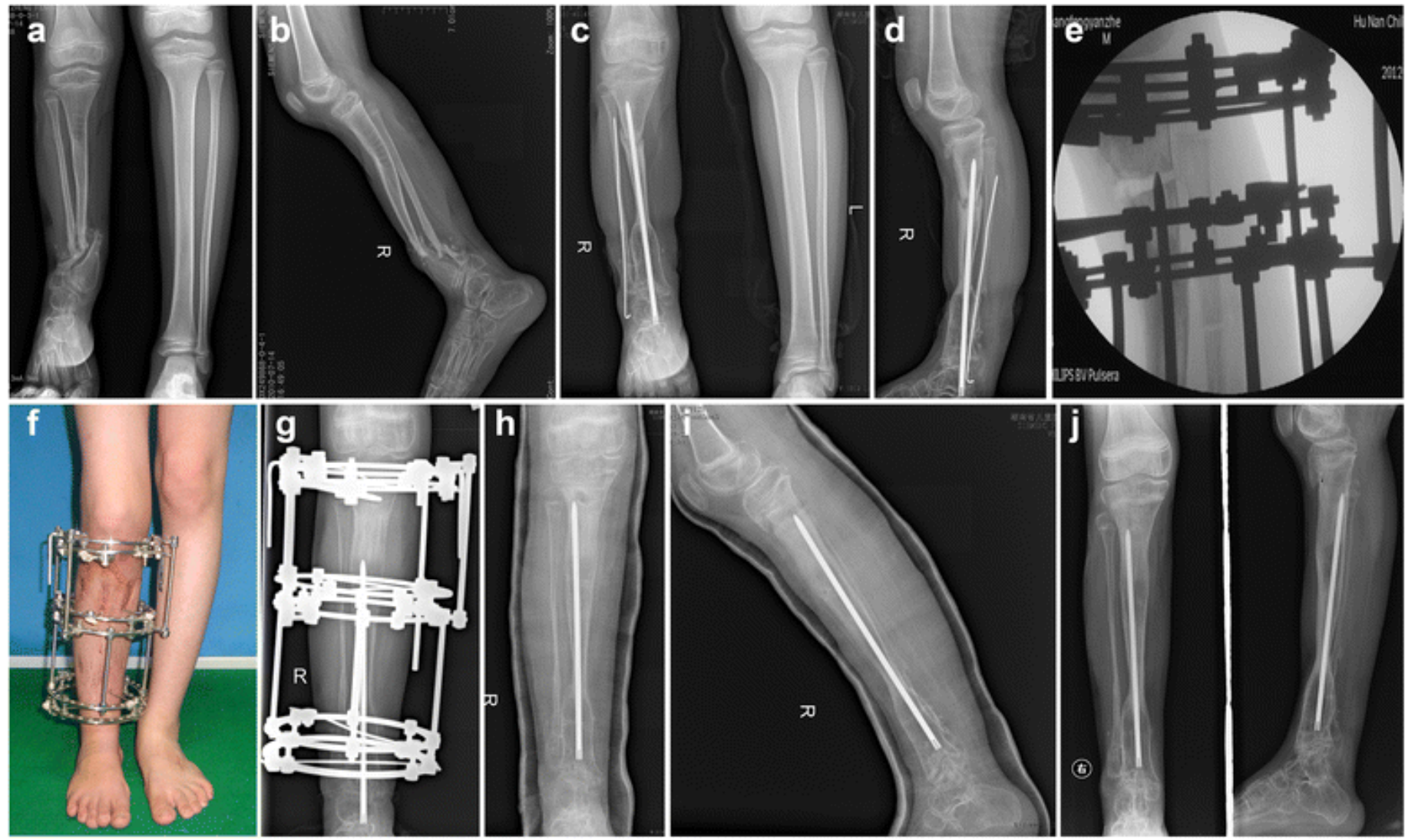

Fig 3

Another representative case (Patient 6). A $14.5 y$ boy with CPT associated with neurofibromatosis type 1 (NF1) had radiological findings of proximal tibial dysplasia $(\mathrm{a}, \mathrm{b})$. Then a combined Ilizarov fixator with intramedullary rodding of the tibia and wrapping autogenic iliac bone graft technique was carried out to manage the pseudarthrosis. And simultaneously a proximal tibial osteotomy was implemented to have access to the normal alignment with the same intramedullary rodding. Two years after the procedure, the radiography showed a primary union of pseudarthrosis but non-union of osteotomy site with LLD of $8 \mathrm{~cm}(\mathrm{c}, \mathrm{d})$. A proximal tibial lengthening and compression of the primary osteotomy site was carried out to equalize the limb length $(\mathrm{e}-\mathrm{g})$. The length gained was $8 \mathrm{~cm}$ with $\mathrm{HI}$ of $68 \mathrm{~d} / \mathrm{cm}$. Then the ex-fixator was removed and the intramedullary rod was pushed into the tibia cavity. The radiography showed both the lengthening segment and the primary osteotomy site were union $(\mathrm{h}, \mathrm{i})$. One year after removal of ex-fixator, $X$ rays showed well corticalization of the lengthening segment with good alignment of tibia, however the ankle joint was stiff with degenerative changes $(\mathrm{j})$

\section{Discussion}

Tibial shortening is a problem which existed before the management of CPT, which was caused by various factors which included bone absorption around the pseudarthrosis area and growth inhibition of distal tibia ${ }^{[7]}$. However, the low union rate of pseudarthrosis compelled the surgeon to concentrate on the bone union other than the sequelae of CPT, such as LLD, abnormal alignment and stiffness of joint. However few studies went ahead and discussed the indication and time window for tibial lengthening. Inan et al. ${ }^{[7]}$ reported the long term sequelae of CPT in 16 patients and found 9 cases with a tibial shortening of $2.6-5.2 \mathrm{~cm}, 3$ cases underwent proximal tibial lengthening but 2 had bone nonunion. They suggested that a contra lateral epiphysiodesis should be carried out to equal the length of lower extremities when the LLD was less than $5 \mathrm{~cm}$, while tibial lengthening could be considered when the LLD was more than $5 \mathrm{~cm}$. Cho et al. ${ }^{[9]}$ introduced their experience of 27 tibial lengthening in 22 patients, some of whom had repeated lengthening. The average length of elongation was $3.7 \mathrm{~cm}$ $(1.0-9.1 \mathrm{~cm})$ and the healing index was $89 \mathrm{~d} / \mathrm{cm}(22 \mathrm{~cm}-$ $280 \mathrm{~d} / \mathrm{cm}$ ). They suggested that if the proximal tibia is not dysplastic and have not been lengthened previously; proximal tibial lengthening by distraction osteogenesis in CPT can be safely performed.

All the tibial lengthening in our study was carried out more than 2 years after initial union of pseudarthrosis with LLD which increased with age. None of the cases had refracture or non-union of distraction gap. We suggest the ideal lengthening timing of tibial shortening for CPT patients should be 2 years after initial union of pseudarthrosis. Proximal tibial lengthening at this point would have no side effect on the pseudarthrosis which already obtained primary union. According to us, tibial shortening over $4 \mathrm{~cm}$ in younger children will cause obvious claudication, and the LLD will increase with age. Postponing the lengthening procedure will lead to more limb length discrepancy to be corrected, longer period of wearing external fixator and higher accompanying risk of complications. We agree with Inan et al. that in most cases tibial lengthening could be considered when the LLD was more than $5 \mathrm{~cm}$. However, for younger children, this indication can be extended to more than $4 \mathrm{~cm}$.

The gold standard of evaluating the quality of bone regeneration is "Healing Index" (HI) ${ }^{[7,14]}$. Compared with Inan [7] reported $\mathrm{HI}$ of $89 \mathrm{~d} / \mathrm{cm}$ and Cho ${ }^{[9]}$ reported 22$280 \mathrm{~d} / \mathrm{cm}$, HI in our series was $63.1 \mathrm{~d} / \mathrm{cm}$. Our result is in accordance of the other studies, which indicated that the tibial lengthening after primary union of CPT had significant difference with tibial lengthening in LLD caused by trauma or other reasons ${ }^{[9,10]}$. Proximal tibial lengthening in CPT 
patients showed different clinical features, characterized by slow regeneration of callus and prolonged fixator wearing period, which may increase the risk of pin tract infection and adjacent joint stiffness. It also had different radiographic feature with poor bone regeneration in the distraction gap, and the callus was scarcely fusiform or cylindrical but lateral or central shaped.

All of our 11 CPT patients underwent proximal tibial lengthening for the first time after primary union. Despite proximal tibial dysplasia in 8 cases, the HI was $63.1 \mathrm{~d} / \mathrm{cm}$ (range, $47-77 \mathrm{~d} / \mathrm{cm}$ ) with no additional procedure(s) to promote bone healing required. In our series, pin tract infection was found in 5 patients $(5 / 11,45.5 \%)$ which was high. We believed this was due to the longer period of fixator wearing. The special preassembled orthosis which was connected to the proximal ring of the Ilizarov fixator could account, in part, for no knee contracture observed. With the help of it, the patient can gradually do active exercise of knee joint. There was one case with deceased motion of ankle joint and one case with stiffness ankle (patient 6), which can be ascribed to previous condition of the ankle joint. But stiffness ankle in patient 4 was mainly because the rod in the centre axis of the ankle.

Despite the complications mentioned above, from the perspective of healing index, the outcome of ours is superior to Inan and Cho's ${ }^{[7,9]}$ which may be caused by the favorable bone condition 2 years or more after primary healing of CPT. We believed that walking with protective orthosis can improve the blood circulation of tibia and increase the density of bone cortex, which will benefit bone callus regeneration. Furthermore, the primary lengthening protocol of our series was starting with $0.5 \mathrm{~mm}$ per day rather than $1.0 \mathrm{~mm}$ described by Cho et al. ${ }^{[9]}$, which may be in accordance with bone callus regeneration of CPT patients.

\section{Conclusions}

We concluded that proximal tibial lengthening after initial union of CPT was effective for management of tibial shortening, however poor bone regeneration with various types of callus from normal, greater healing index and prolonged fixator wearing was seen. We recommended that when the limb length discrepancy (LLD) was more than $4 \mathrm{~cm}$ in younger children after primary union of CPT, tibia lengthening could be considered.

\author{
Abbreviations \\ CPT: Congenital pseudarthrosis of the tibia \\ LLD: Limb length discrepancy \\ HI: Healing index
}

\section{References}

1. Hefti F, Bollini G, Dungl P, Fixsen J, Grill F, Ippolito E et al. Congenital pseudarthrosis of the tibia: history, etiology, classification, and epidemiologic data. J Pediatr Orthop B. 2000; 9(1):11-5.

2. Thabet AM, Paley D, Kocaoglu M, Eralp L, Herzenberg JE, Ergin ON. Periosteal grafting for congenital pseudarthrosis of the tibia: a preliminary report. Clin Orthop Relat Res. 2008; 466(12):2981-94.

3. Shah H, Doddabasappa SN, Joseph B. Congenital pseudarthrosis of the tibia treated with intramedullary rodding and cortical bone grafting: a follow-up study at skeletal maturity. J Pediatr Orthop. 2011; 31(1):79-88.

4. Pannier S, Pejin Z, Dana C, Masquelet AC, Glorion C. Induced membrane technique for the treatment of congenital pseudarthrosis of the tibia: preliminary results of five cases. J Child Orthop. 2013; 7(6):477-85.

5. Khan T, Joseph B. Controversies in the management of congenital pseudarthrosis of the tibia and fibula. Bone Joint J. 2013; 95(8):1027-34.

6. Pannier S. Congenital pseudarthrosis of the tibia. Orthop Traumatol Surg Res. 2011; 97(7):750-61.

7. Inan M, El Rassi G, Riddle EC, Kumar SJ. Residual deformities following successful initial bone union in congenital pseudarthrosis of the tibia. J Pediatr Orthop. 2006; 26(3):393-9.

8. Kristiansen LP, Steen H, Terjesen T. Residual challenges after healing of congenital pseudarthrosis in the tibia. Clin Orthop Relat Res. 2003; 414:228-37.

9. Cho TJ, Choi IH, Lee KS, Lee SM, Chung CY, Yoo WJ et al. Proximal tibial lengthening by distraction osteogenesis in congenital pseudarthrosis of the tibia. $\mathbf{J}$ Pediatr Orthop. 2007; 27(8):915-20.

10. Vlad C, Gavriliu TS, Georgescu I, Dan D, Parvan A, Burnei G. Bone transport with the lengthening through the physis in patients having congenital pseudarthrosis of tibia - short-term results. J Med Life. 2013; 6(3):266-71.

11. Mathieu L, Vialle R, Thevenin-Lemoine C, Mary P, Damsin JP. Association of Ilizarov's technique and intramedullary rodding in the treatment of congenital pseudarthrosis of the tibia. J Child Orthop. 2008; 2(6):449-55 